\title{
Thymidylate Synthase and Dihydropyrimidine Dehydrogenase Are Upregulated in Pancreatic and Biliary Tract Cancers
}

\author{
Mitsugi Shimoda Tokihiko Sawada Keiichi Kubota \\ Second Department of Surgery, Gastroenterological Surgery, Dokkyo Medical University School of Medicine, \\ Mibu, Japan
}

\section{Key Words}

Thymidylate synthase $\cdot$ Dihydropyrimidine dehydrogenase $\cdot$ Pancreatic cancer $\cdot$ Biliary tract cancer

\begin{abstract}
Purpose: To investigate a rationale of pancreatobiliary cancers, thymidylate synthase (TS) and dihydropyrimidine dehydrogenase (DPD) concentrations in cancer tissues were evaluated. Patients and Methods: Twenty-nine patients with pancreatic cancer (PC) and 43 with biliary tract cancer (BTC; 27 cases of bile duct cancer, 8 of gallbladder cancer and 8 of cancer of the papillary area) were included. ELISA was used to assess the concentrations of TS and DPD in cancerous and noncancerous tissues. All 82 patients were followed up at least 12 months after surgery. We compared patient survivals between the 2 groups, taking TS and DPD cutoff as median values, high versus low DPD and high versus low TS in the cancerous tissue of PC and BTC patients. Results: As for PC, DPD and TS concentrations were significantly higher in cancerous than in noncancerous tissues ( $p=0.016$ vs. 0.036). In BTC, TS concentration was higher in cancerous than in noncancerous tissue $(p=0.0001)$. Patients with low TS and DPD concentrations had a tendency to have a better disease-free survival than those with high TS and DPD concentrations in PC groups; especially, BTC patients' diseasefree survival with high TS was significantly longer than that
\end{abstract}

with low TS ( $p=0.0083)$. Discussion: TS and DPD levels had a tendency to be high in PC and BTC cancerous tissues. High TS in the cancerous tissue was associated with better disease-free survival in BTC.

Copyright $\odot 2009$ S. Karger AG, Basel

\section{Introduction}

Pancreatic cancer (PC) is the fifth most common cause of death in Japan (mortality: about 20,000 people in 2002) and the incidence is increasing worldwide [1-3]. Particularly, the median survivals of patients with unresectable tumors due to local extension and those with distant metastasis were 6-10 and 3-6 months, respectively. The prognosis is also poor even for patients with resectable tumors and, compared with other types of cancer, the 5year survival rate is only $13 \%$ [4].

On the other hand, about 16,000 people die annually of biliary tract cancer (BTC), accounting for the sixth largest number of cancer deaths. However, BTC is often found in an advanced stage since it rarely produces symptoms, and the prognosis is poor, with high rates of recurrence after resection [1-3].

Drug-metabolizing enzymes, thymidylate synthase (TS) and dihydropyrimidine dehydrogenase (DPD) are frequently upregulated in cancer tissues particularly in

\section{KARGER}

Fax +4161306 1234 E-Mail karger@karger.ch www.karger.com

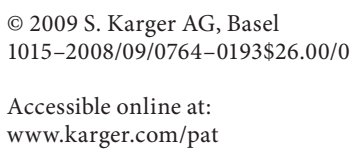

Mitsugi Shimoda, MD, PhD

Department of Gastroenterological Surgery

Dokkyo Medical University School of Medicine

Mibu, Tochigi 321-0293 (Japan)

Tel. +81 28287 2158, Fax +81 28286 6317, E-Mail mshimoda@dokkyomed.ac.jp 
colon and gastric cancer [5-7]. After entering into cells, 5-fluorouracil (5-FU) is partly degraded to 2-fluoro$\beta$-alanine by DPD, and undegraded intact 5 -FU are metabolized to 5-fluoro-deoxyuridine monophosphate (FdUMP), FdUMP suppresses the activity of TS, resulting in the inhibition of DNA synthesis. Thus, the upregulation of DPD and TS lets cancer cells be resistant to conventional 5-FU anticancer drugs. To improve the effect of 5-FU anticancer drugs, suppression of intratumoral DPD has been demanded.

To date, there have been no reports describing whether TS and DPD are upregulated in PC and BTC. We hypothesized that cancerous tissue levels of TS and DPD affect prognostic factors for disease-free survival (DFS) in PC and BTC.

\section{Patients and Methods}

From July 2003 to March 2006, 113 patients with PC or BTC visited our department. Among them, we collected samples from 29 with PC and 43 with BTC.

Patients suspected of having PC or BTC were examined by computed tomography, ultrasonography and angiography, and tumor markers (carbohydrate antigen: CA19-9, CEA) were measured. After obtaining the study protocol approval from the Institutional Review Board, patients with resectable malignant pancreatic and biliary tract tumors, who gave their written informed consent, were enrolled for this study. Surgical resection was contraindicated for patients with arterial invasion and distant metastasis of the tumor detected on the preoperative diagnostic images. Patients with tumors that were initially considered resectable based on the results of imaging procedures, but found to be unresectable at laparotomy (peritoneal dissemination, liver metastasis, arterial and portal invasion), were not enrolled. None of the 82 patients received any kinds of adjuvant chemotherapy agents in this study period. Cancerous and noncancerous tissues were obtained in the main tumor and close to the surgical margin.

The measurement of TS and DPD levels by ELISA was delegated to Taiho Pharmaceutical Co. Ltd. The assays have been described in detail previously [7]. Briefly, plastic trays coated with TS or DPD antibodies were allowed to react with the homogenized specimens. The specimens were then allowed to react with secondary coloring antibodies, and color-fixing agents were added. Fluorescence levels were quantified by a sandwich technique.

Actual concentrations of TS and DPD in cancerous and noncancerous tissues were measured. TS and DPD ratios were calculated by dividing concentration in noncancerous tissue with that in cancerous tissue. A ratio below 1.0 means that TS and DPD are upregulated in cancerous tissue.

We evaluated DFS for all 82 patients and compared DFS for high versus low DPD and high versus low TS in the cancerous tissue in PC and BTC patients. Overall survival is not well provided, because more than $50 \%$ of recurrent patients received gemcitabine treatment. TS and DPD levels had no direct effect for patients' overall survival in this retrospective analysis. The cutoff level of
Table 1. DPD and TS in patients

\section{$P C(n=29)$}

Resected cases: 15 (12 with pancreatic head cancer and 3 with pancreatic body and tail cancer)

Measurable DPD in the tissue samples: 15 patients

Measurable TS in the tissue samples: 8 patients Nonresected cases: 14 (9 with pancreatic head cancer and 50 with pancreatic body and tail cancer)

\section{Gallbladder and bile duct cancer $(n=43)$}

Resected cases: 33 (20 with bile duct cancer, 8 with cancer of the papilla and 5 with gallbladder cancer)

Measurable DPD in the tissue samples: 30 patients

Measurable TS in the tissue samples: 27 patients Nonresected cases: 10 (7 with bile duct cancer and 3 with gallbladder cancer) low and high levels was a median value. All 82 patients were followed up at our institution at least for 2 years after surgery.

Statistical Analysis

Mann-Whitney U test or Wilcoxon's test was used for withingroup comparisons. Survival analysis was performed by the Kaplan-Meier analysis and log-rank test to compare patient's survival. A probability value of $\mathrm{p}<0.05$ was considered to indicate statistical significance.

\section{Results}

In the 29 patients with $\mathrm{PC}$, tumors could be resected in 15 patients (table 1). DPD was measurable in tumor tissues in 15 patients and TS in 8 patients. We measured the median values of DPD and TS in cancerous and noncancerous tissues. Median values of DPD in cancerous and noncancerous tissues were 121.0 (range 38.9-465.0) and $76.3 \mathrm{ng} / \mathrm{mg}$ protein (range 11.1-180.8), respectively. Also, median values of TS in cancerous and noncancerous tissues were 7.0 (range $0.6-11.0$ ) and $1.7 \mathrm{ng} / \mathrm{mg}$ protein (range 0.3-2.6), respectively. In cancerous tissues, DPD and TS concentrations were significantly higher than in normal tissues ( $\mathrm{p}=0.016$ : DPD, fig. 1a; $\mathrm{p}=0.036$ : TS, fig. 1b).

Among the 43 BTC patients, there were 27 with bile duct cancer, 8 with gallbladder cancer and 8 with cancer of the papilla of Vater. Tumors could be resected in 33 patients (table 1). DPD and TS levels were measurable in tumor tissues in 30 and 27 patients, respectively. We also measured the median values of DPD and TS concentra- 
Fig. 1. a, b In patients with PC, DPD and TS expression in cancerous tissue was significantly higher.
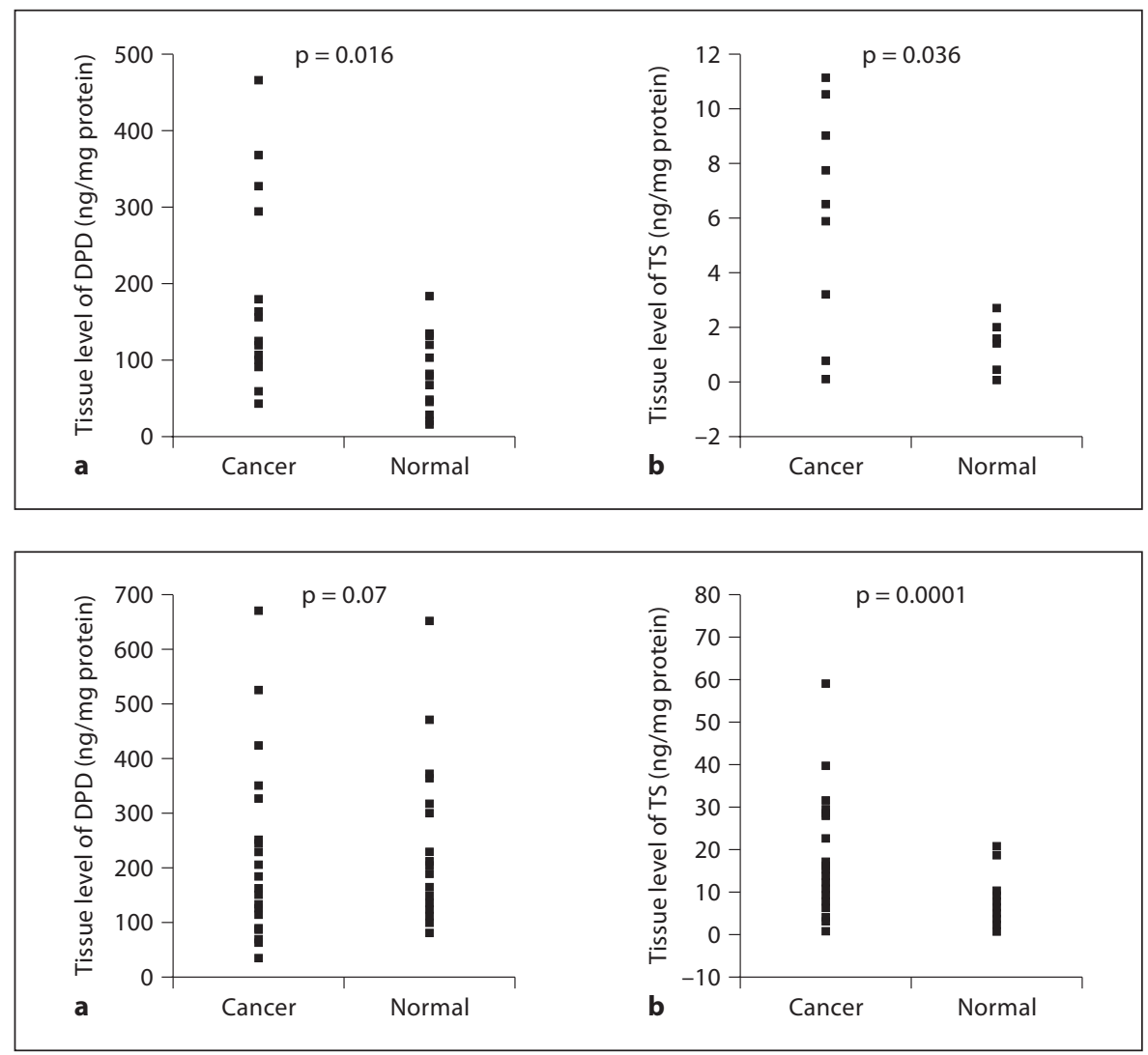

Fig. 2. a, b In patients with BTC, the expression of DPD did not differ significantly between cancerous and normal tissues, but the difference in TS expression was statistically significant.

Fig. 3. a, b In the PC and BTC groups, the TS and DPD ratio (normal tissue/cancerous tissue) were within 1.0.
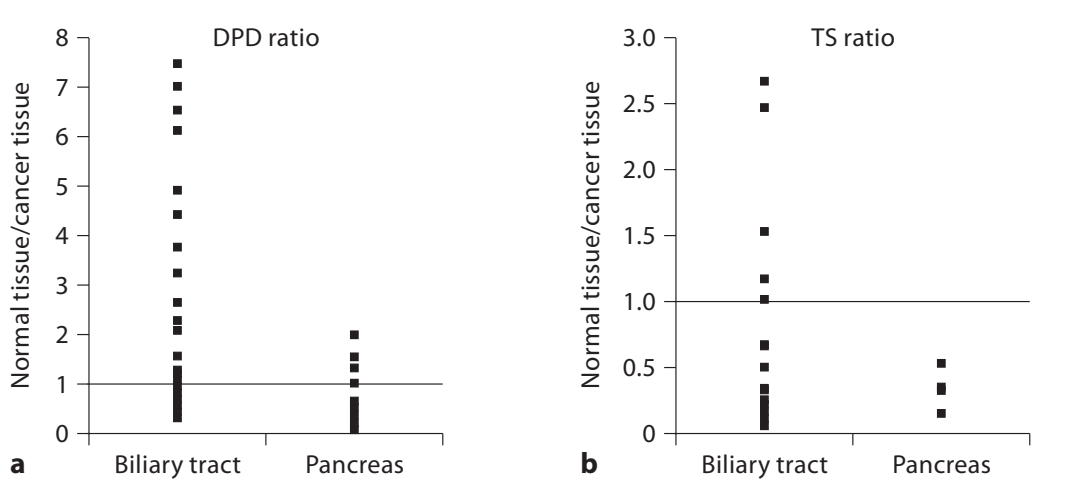

tions in cancerous and noncancerous tissues. Median values of DPD in cancerous and noncancerous tissues were 145.2 (range 30.1-665.4) and $134.9 \mathrm{ng} / \mathrm{mg}$ protein (range 76.3-644.7), respectively. Also, median values of TS in cancerous and noncancerous tissues were 13.4 (range 2.3-212.7) and $4.3 \mathrm{ng} / \mathrm{mg}$ protein (range 0.4-20.0), respectively. DPD expression did not differ significantly between cancerous and noncancerous tissues $(\mathrm{p}=0.07$ : fig. 2a); but the difference in TS expression was statistically significant $(\mathrm{p}=0.0001$ : fig. $2 \mathrm{~b})$.

TS and DPD Are Upregulated in Pancreatic and Biliary Tract Cancers
In PC and BTC, the DPD and TS ratio (normal tissue/ cancerous tissue) was less than 1.0 (fig. 3a and b), indicating that DPD and TS were upregulated in cancerous tissues.

Concerning DFS, in the PC group, 8 of 15 patients had recurrent tumor and 5 of 8 patients underwent gemcitabine treatment. The median values of TS and DPD concentrations in patients with PC were 7.0 (range 0.611.0 ) and $121.0 \mathrm{ng} / \mathrm{mg}$ protein (range 38.9-465.0) in cancerous tissue, respectively. Low TS patients $(<7.0 \mathrm{ng} / \mathrm{mg}$

Pathobiology 2009;76:193-198 


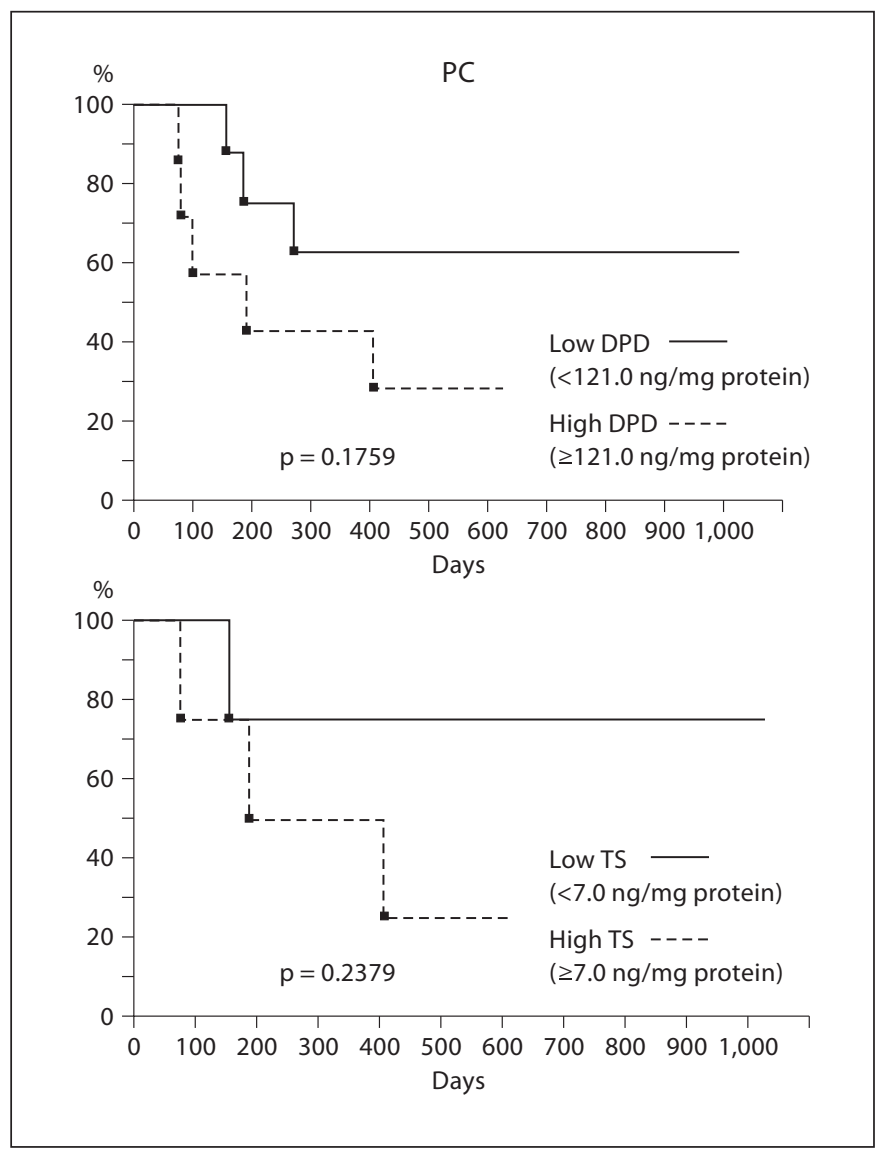

Fig. 4. In the PC group, high DPD DFS rate was $62.5 \%$ and low DPD DFS was $42.95 \%$ at 1 year, there was no significant difference; high TS DFS rate was $50 \%$ and low TS DFS was $75 \%$ at 1 year, there was no significant difference.

protein) had a tendency to have a better DFS rate than high TS patients ( 75 and $50 \%$ at 1 year, respectively, $\mathrm{p}=$ 0.237 ; fig. 4). Low DPD patients ( $<121.0 \mathrm{ng} / \mathrm{mg}$ protein) also had a tendency to have a better DFS rate than high DPD patients (62.5 and $42.95 \%$ at 1 year, respectively, $\mathrm{p}=$ 0.175; fig. 4). In the BDC group, 7 of 27 patients had recurrent tumor, while 4 of 7 patients underwent gemcitabine treatment. The median values of TS and DPD concentrations in patients with BDC were 13.4 (range 2.3-212.7) and $145.2 \mathrm{ng} / \mathrm{mg}$ protein (range 30.1-665.4) in cancerous tissue, respectively. The survival of high TS $(<13.4 \mathrm{ng} / \mathrm{mg})$ patients was significantly better than that of low TS patients (91.7 and $51.3 \%$ at 1 year, respectively, $\mathrm{p}=0.0083$; fig. 5). The DFS rate of high DPD $(<145.2) \mathrm{pa}-$ tients had a tendency to be better than that of low DPD patients (78.6 and $73.3 \%, \mathrm{p}=0.831$; fig. 5).

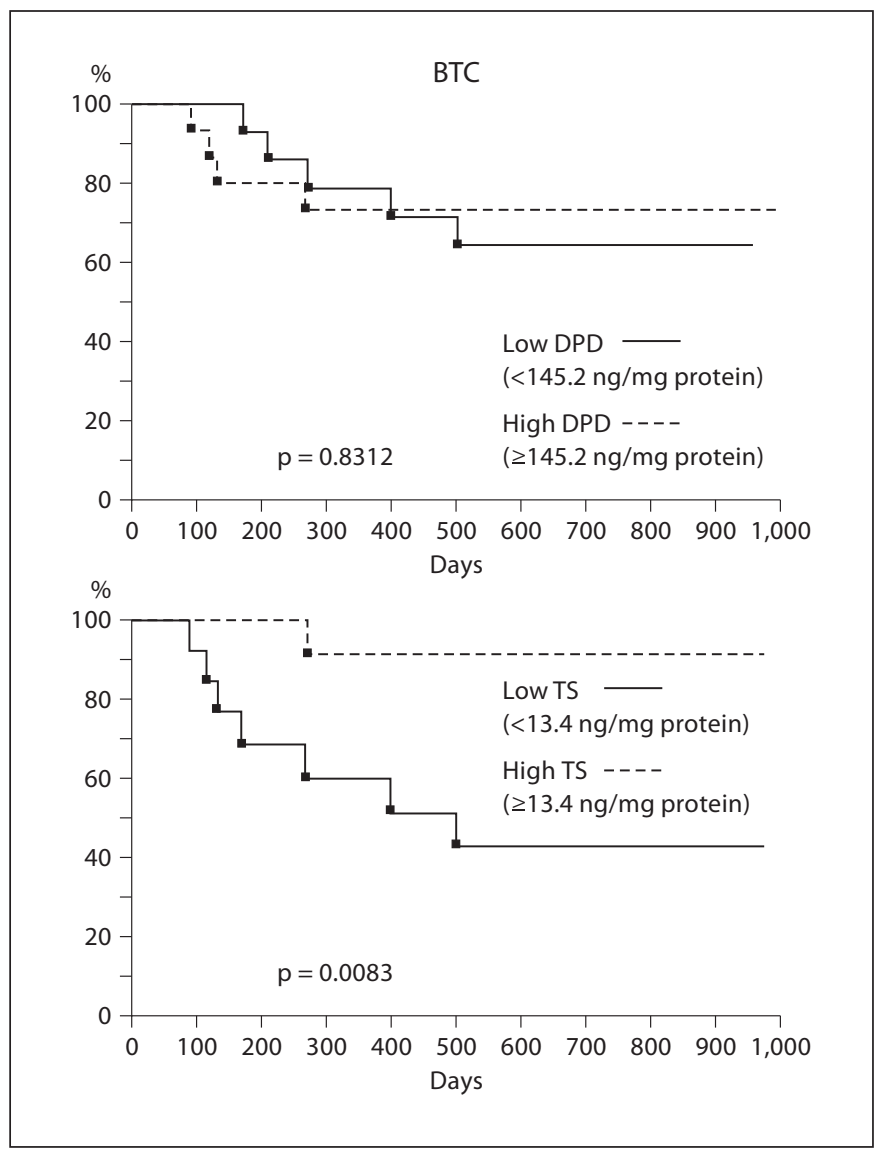

Fig. 5. In the BTC group, high TS DFS rate was $91.7 \%$ and low TS DFS was $51.3 \%$ at 1 year, there was significant difference; high DPD DFS rate was $78.6 \%$ and low DPD DFS was $73.3 \%$ at 1 year, there was no significant difference.

\section{Discussion}

There are few reports on DPD and TS concentrations, which are 5-FU metabolism-related enzymes, in PC and BTC tissues $[8,9]$. In this study, we compared DPD and TS concentrations in PC or BTC tissues with those in normal tissues for the first time, and found that TS and DPD concentrations were higher in cancerous than in normal tissues. Figure 3 clearly indicates that TS and DPD concentrations had a tendency to be higher in cancerous than in normal tissues in most patients. This upregulation of TS and DPD in cancerous tissue might result in the tumor's resistance to conventional 5-FU derivatives because conventional 5-FU derivatives would be easily metabolized to inactivate substances, resulting in less FdUMP and, subsequently, a small part of TS could be combined by FdUMP. 
Since the FDA approval of gemcitabine for patients with PC was announced in 1996, 50 or more phase I, II and III studies on gemcitabine-based combination chemotherapy and gemcitabine alone have been reported $[10,11]$. However, the average survival has been reported to be 5.7 months for gemcitabine alone and 7.4 months for combination chemotherapy [10-16]. As for BTC, there have been no reports showing that chemotherapy or radiation therapy has remarkably improved the prognosis. Although use of gemcitabine-based anticancer drug therapy has been increasing, the reported response rate is only relatively good at $15-30 \%$ [17]. The next step should be the advent of a new anticancer drug that is effectively used with or instead of gemcitabine.

In our study, we paid attention to the effect of 5-FU anticancer drugs as an alternative to gemcitabine. To analyze the effect of 5-FU anticancer drugs, we measured protein levels of DPD and TS as well as drug-metabolizing enzymes in the tissues of PC and BTC. Fukushima et al. [7] measured TS and DPD levels in 5 different cancerous tissues, such as gastric, colorectal, breast, lung and head as well as neck cancers, using ELISA methods. The total number of samples was 13,131. Median values of TS and DPD were 22.1 (range 10.9-30.6) and $135.4 \mathrm{ng} / \mathrm{mg}$ protein (range 111.2-264.0), respectively. TS levels of PC and BTC in our patients were slightly lower than those reported by Fukushima et al. [7], while DPD levels were almost similar to those.

TS has been considered useful for predicting response to 5-FU anticancer drugs, leading to the hypothesis that tumors with low TS level are sensitive to 5-FU. Further- more, high DPD level in the cancerous tissue is considered to accelerate the decomposition of the conventional 5-FU.

Ichikawa et al. $[18,19]$ reported that survival was significantly better in patients with gastric cancer or colon cancer with low TS and low DPD mRNA expression than in patients with high TS and high DPD mRNA expression. Patients with low level of DPD were considered to be good responders to 5 -FU anticancer drugs ( $\mathrm{p}=0.0001)$. Kuromachi et al. [20] also reported that DPD mRNA revel in PC was significantly higher than in colorectal and gastric cancer. 5-FU good responders had lower level of DPD mRNA expression than nonresponders $(\mathrm{p}=0.012)$. In this study, DFS rates of PC tended to be better in low than in high DPD in cancerous tissue, and tended to be better in low than in high TS in cancerous tissue, however, there were no significant differences. In the mean time, DFS rate of patients with BTC with high TS cancerous tissue was significantly better than that of patients with low TS concentrations. Although DFS rates of BTC tended to be better in patients with high DPD in cancerous tissue than in patients with low DPD, there was no significant difference. It was suggested that high level of TS and DPD in cancerous tissue were good prognostic factors for DFS. These results suggested that 5-FU treatment is a different way for PC and BTC patients; prospective study is needed.

In conclusion, we demonstrated that TS and DPD are upregulated in cancerous tissues in pancreatobiliary cancer for the first time. The high levels of TS in BTC cancerous tissue may be good prognostic factors for DFS.

\section{References}

1 Demographic statistics of the Ministry of Health, Labour and Welfare. http://www. mhlw.go.jp/toukei/saikin/hw/jinkou/kakutei03/index.html.

2 Halpern MT, Ward EM, Pavluck AL, Schrag NM, Bian J, Chen AY: Association of insurance status and ethnicity with cancer stage at diagnosis for 12 cancer sites: a retrospective analysis. Lancet Oncol 2008;3:189-191.

$\checkmark 3$ Henson DE, Albores-Saavedra J, Corle D: Carcinoma of the extrahepatic bile ducts. Histologic types, stage of disease, grade, and survival rates. Cancer 1992;70:1498-1501.

4 Seiki Matsuno (Japan Pancreas Society), Pancreatic Cancer Register Committee of Japan Pancreas Society: Summary of 20 years. Suizou 2003;18:97-169.

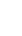

Yamamoto S, Kubota K: Level of 5-fluorodeoxyuridine $5^{\prime}$-monophosphate in cancerous tissue in patients with gastric cancer under preoperative administration of TS-1: a preliminary study. J Exp Clin Cancer Res 2005; 24:457-462.

6 Kurebayashi J, Yamamoto Y, Udagawa K, Okubo S, Fukushima M, Sonoo H: Establishment of enzyme-linked immunosorbent assays for thymidylate synthase and dihydropyriminide dehydrogenase in cancer tissues. Oncol Rep 2004;11:973-979.

7 Fukushima M, Fukui Y, Sugimoto Y, Oka T, Nagayama S: Population study of thymidylate synthase, dihydropyrimidine dehydrogenase and orotate phosphoribosyltransferase in patients with solid tumors. Proc AACR Meet Abstr 2006;47:854-855.
8 Nagakawa T, Kayahara M, Ohta T, Kitagawa H, Mikami K, Kurata T, Otsuji S: Dihydropyrimidine dehydrogenase activity in human pancreatic tumor tissues. Cancer Invest 2000;18:516-520.

9 Kuramochi $\mathrm{H}$, Hayashi K, Uchida K, Miyakura S, Shimizu D, Vallbohmer D, Park S, Danenberg KD, Takasaki K, Danenberg PV: 5-fluorouracil-related gene expression levels in primary colorectal cancer and corresponding liver metastasis. Int J Cancer 2006; 119:522-526. 
10 Heinemann V, Quietzsch D, Gieseler F, Gonnermann M, Schonekas H, Rost A, Neuhaus $\mathrm{H}$, Haag C, Clemens M, Heinrich B, VehlingKaiser U, Fuchs M, Fleckenstein D, Gesierich W, Uthgenannt D, Einsele H, Holstege A, Hinke A, Schalhorn A, Wilkowski R: Randomized phase III trial of gemcitabine plus cisplatin compared with gemcitabine alone in advanced pancreatic cancer. J Clin Oncol 2006;24:3946-3952.

-11 Jordan DB, Paul C, James PT, et al: Phase III study of gemcitabine in combination with fluorouracil versus gemcitabine alone in patients with advanced pancreatic carcinoma: Eastern cooperative oncology group trial E2297. J Clin Oncol 2002;20:3270-3275.

-12 Bramhall SR, Rosemurgy A, Brown PD, et al: Marimastat as first-line therapy for patients with unresectable pancreatic cancer. J Clin Oncol 2001;19:3447-3455.

-13 Howard AB, Malcolm JM, John A, et al: Improvement in survival and clinical benefit with gemcitabine as first-line therapy for patients with advanced pancreatic cancer. J Clin Oncol 1997; 15:2403-2413.
14 Van Cutsem E, van de Velde H, Karasek P, et al: Phase III trial of gemcitabine plus tipifarnib compared with gemcitabine plus placebo in advanced pancreatic cancer. J Clin Oncol 2004;22:1430-1438.

15 Moore MJ, Hamm J, Dancey J, et al: Comparison of gemcitabine versus the matrix metalloproteinase inhibitor BAY 12-9566 in patients with advanced or metastatic adenocarcinoma of the pancreas: a phase III trial of the National Cancer Institute of Canada clinical trials group. J Clin Oncol 2003;21: 3296-3302.

16 Okada S, Ueno H, Okusaka T, et al: Phase I trial of gemcitabine in patients with advanced pancreatic cancer. Jpn J Clin Oncol 2001;31:7-12.

17 Ishii H, Okusaka T, Funakoshi A: Treatment strategy for unresectable biliary tract cancer - focus on the results of joint multicenter phase II studies of gemcitabine hydrochloride. Jpn Soc Gastroenterol 2005;102:A79.
18 Ichikawa W, Takahashi T, Suto K, Yamashita T, Nihei Z, Shirota Y, Shimizu M, Sasaki Y, Hirayama R: Thymidylate synthase predictive power is overcome by irinotecan combination therapy with S-1 for gastric cancer. $\mathrm{Br}$ J Cancer 2004;91:1245-1250.

19 Ichikawa W, Uetake H, Shirota Y, Yamada H, Nishi N, Nihei Z, Sugihara K, Hirayama R: Combination of dihydropyrimidine dehydrogenase and thymidylate synthase gene expressions in primary tumors as predictive parameters for the efficacy of fluoropyrimidine-based chemotherapy for metastatic colorectal cancer. Clin Cancer Res 2003;9: 786-791.

20 Kuramochi H, Hayashi K, Uchida K, Nakajima G, Hatori T, Danenberg KD, Danenberg PV, Yamamoto M: Thymidylate synthase, dihydropyrimidine dehydrogenase, orotate phosphoribosyltransferase mRNA and protein expression levels in solid tumors in large scale population analysis. Cancer Chemother Pharmacol 2008;63:85-89. 